\title{
Manejo integrado en el cultivo de anón (Annona squamosa L.)
}

\section{Integrated management of sugar apple (Annona squamosa L.)}

EUGENIO DE JESÚS GUERRERO' GERHARD FISCHER ${ }^{2}$
Anón proveniente de Apulo (Cundinamarca) envuelto en papel de seda y empacado en caja de plástico.

Foto: G. Fischer

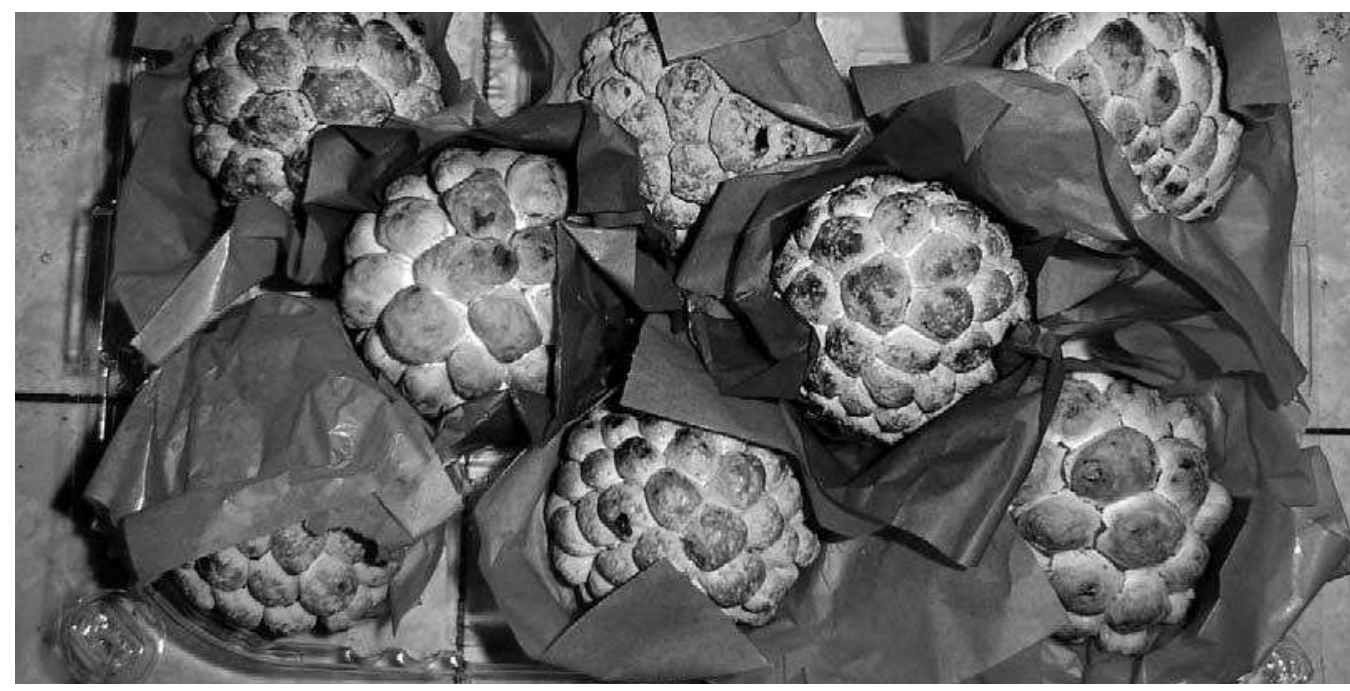

\section{RESUMEN}

Existen varias zonas productoras de anón en Colombia que cuentan con las condiciones agroclimáticas óptimas para el desarrollo de este frutal; pero debido al deficiente manejo del cultivo en el país, es muy baja la producción y rentabilidad del mismo. Este trabajo se realizó con el objetivo de implementar un manejo integrado a través de una amplia revisión bibliográfica y observaciones hechas en la granja "El Ensueño" del municipio de Apulo, Cundinamarca. El anón proviene de las regiones tropicales de Centroamérica o las Antillas; es un árbol semicaducifolio de porte bajo de 3 a $7 \mathrm{~m}$ de altura. Crece por debajo de $1.000 \mathrm{msnm}$, con temperaturas de 10 a $20^{\circ} \mathrm{C}$ como mínimas y de 22 a $28^{\circ} \mathrm{C}$ como máximas. Requiere de 750 a $1.000 \mathrm{~mm}$ de lluvia anual y humedad relativa superior a $60 \%$. Es poco exigente en tipo de suelo. El cuajamiento de frutos y la productividad del cultivo aumentan con el uso de polinización artificial o con la liberación de insectos polinizadores. Dentro de las plagas más importantes se encuentra la avispa perforadora de las semillas (Bephratelloides maculicollis Cam.) y la polilla perforadora del fruto (Cerconota anonella Sepp.). Las enfermedades más limitantes son la antracnosis (Colletotrichum gloeosporioides) que ataca hojas, flores y frutos, y la mancha blanca del follaje (Cercospora annonae). El fruto presenta un comportamiento climatérico.

Ingeniero Agrónomo, Facultad de Agronomía, Universidad Nacional de Colombia, Bogotá (Colombia). guerreroun@yahoo.com

2 Profesor Asociado, Facultad de Agronomía, Universidad Nacional de Colombia, Bogotá (Colombia).

gfischer@unal.edu.co 
Palabras clave adicionales: agroecología, polinización artificial, antracnosis, Bephratelloides macullicolis, fruto climatérico.

\section{ABSTRACT}

There are several producing zones of sugar apple in Colombia, which count on ideal agro climatic conditions to develop this fruit tree; though, due to the deficient management, the production and profitability are very low. This project was aiming to implement an integrated management through a wide literature review and observations realized at the farm "El Ensueño" in Apulo (Cundinamarca); central part of Colombia. Sugar apple is native from the tropical regions of Central America or the Antilles; it is a small semi-deciduous tree growing from 3 to $7 \mathrm{~m}$ height. This crop grows below 1,000 $\mathrm{m}$ above sea level, with temperatures from minimum 10$20^{\circ} \mathrm{C}$ and to $22-28^{\circ} \mathrm{C}$ max. It requires 750 to $1,000 \mathrm{~mm}$ of annual rainfall and relative humidity higher than $60 \%$. It is slightly demanding soil requirements. Fruit set and productivity increase with the use of artificial pollination or liberation of pollinators. Inside the most important pest insects there are the wasp seed borer (Bephratelloides maculicollis Cam.) and the moth fruit borer (Cerconota anonella Sepp.). The most restricting diseases are the anthracnose (Colletotrichum gloeosporioides) attacking leaves, flowers and fruits and the white spot of foliage (Cercospora annonae). The fruit presents a climacteric behavior.

Additional key words: agro ecology, artificial pollination, anthracnose, Bephratelloides macullicolis, climacteric fruit.

Fecha de recepción: 06-03-2007

Aprobado para publicación: 28-11-2007
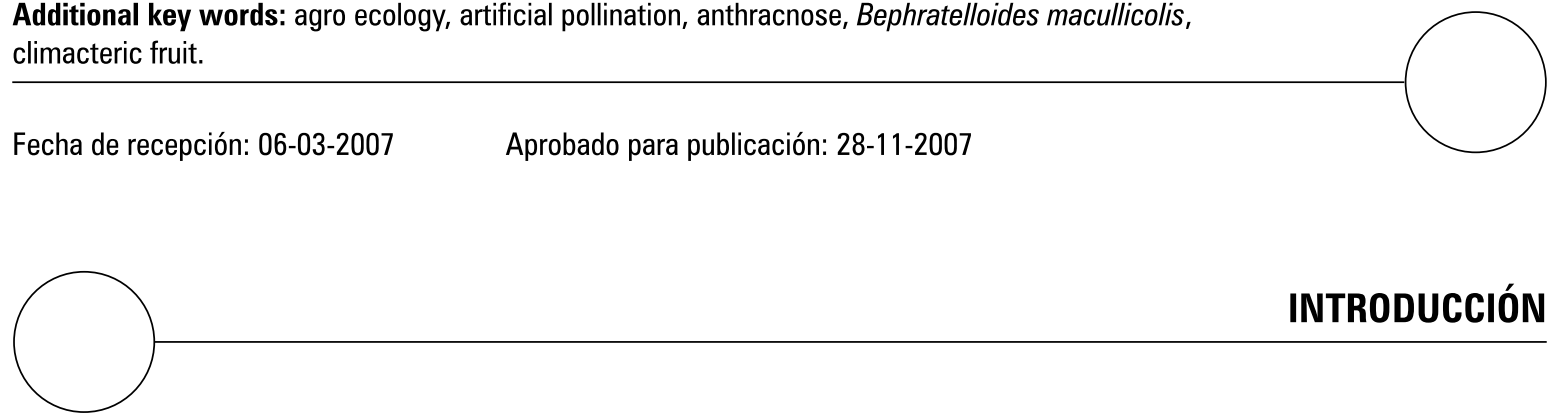

El anón (Annona squamosa L.) pertenece a la familia de las anonáceas, la cual alberga cerca de 2.500 especies en 140 géneros. El género Annona es uno de los más importantes ya que posee 150 especies dentro de las que se encuentra además del anón, otras de interés comercial como la guanábana (Annona muricata L.), la chirimoya (Annona cherimola Mill.) y más recientemente el híbrido atemoya (A. squamosa x $A$. cherimola) proveniente del cruce entre anón y chirimoya. Estas frutas han ganado una gran aceptación entre los consumidores y juntas podrían constituirse en un grupo de frutas con características muy apetecibles y con grandes oportunidades en el sector agroindustrial.
Pese al gran potencial que representa esta especie frutícola, es poco el manejo que se le da al cultivo, debido a que no se conocen las tecnologías de producción. Además, la expansión del cultivo está limitada por la poca información existente sobre la especie, principalmente en relación a aspectos biológicos, botánicos y agronómicos (Pinto y Genú, 1984).

Debido al deficiente manejo del cultivo en las zonas productoras (árboles sin podar, propagación solamente por semillas, fertilización deficiente, insuficiente irrigación, escasa polinización, falta de identificación y manejo de los problemas fitosanitarios, desconocimiento de los índices 
de madurez, mal manejo poscosecha), se realizó este trabajo con el objetivo de implementar un manejo integrado a través de una exhausta revisión bibliográfica, observaciones en campo y primeros ensayos en la granja "El Ensueño" del municipio de Apulo, Cundinamarca ( $4^{\circ} 31^{\prime}$ de latitud norte y $74^{\circ} 36^{\prime}$ de longitud oeste. Altitud $420 \mathrm{msnm}$, temperatura promedio anual $26,8^{\circ} \mathrm{C}$ y precipitación anual de $1.100 \mathrm{~mm}$. Según la clasificación de Holdridge, pertenece a bosque seco tropical (bs-T).

\section{USOS}

Los frutos se consumen principalmente frescos, ya que tienen un sabor cremoso y dulce; son muy nutritivos, ricos en azúcares, proteínas y fósforo, con una pulpa muy digestiva, por lo que se recomienda en la dieta de niños y ancianos. También se utilizan en postres y en la elaboración de jugos, refrescos, zumos, sorbetes, vinos, helados y bebidas espirituosas (Navarro, 2001).

La decocción de las flores se usa para combatir el reumatismo mediante baños en la frente (Hoyos, 1989). El té obtenido de las raíces sirve como purgante, mientras que el que se hace con las hojas es ligeramente laxante. El fruto verde, muy astringente, es empleado contra la diarrea (Leal, 1990).

El fruto verde desecado, las semillas y las hojas pulverizadas se utilizan como insecticidas. La cáscara, las hojas, los tallos y las semillas contienen fibras, aceites y varios alcaloides, los cuales tienen aplicaciones insecticidas, fungicidas, medicinales e industriales (Rajput, 1985; Morton, 1987).

\section{ORIGEN Y DISTRIBUCIÓN GEOGRÁFICA}

No se conoce con exactitud el lugar de origen del anón. Antes se creía que era nativo de la India, pero datos históricos y filológicos tienden a confirmar que es de origen americano (Rajput,
1985). Probablemente es nativo de las regiones tropicales de Centroamérica o las Antillas (Morton, 1987).

Es la especie más ampliamente distribuida del género Annona en el mundo. Se cultiva en la zona tropical de Suramérica, en el sur de México, el occidente de la India, Bahamas, Bermuda, y en el sur de la Florida. En las zonas cálidas tropicales de América, Jamaica, Puerto Rico, Barbados, el sur de la India y en las regiones secas del norte de Queensland, Australia, se encuentra en forma silvestre en praderas y bosques (Rajput, 1985; Morton, 1987; Hoyos, 1989).

El cultivo es extenso en India, mientras que en Brasil es uno de los frutos más importantes, siendo llamativo en los mercados de Bahía. En Colombia se encuentra en la Costa Atlántica y en las zonas secas de los valles interandinos, en los departamentos de Valle, Caldas, Huila, Tolima, Cundinamarca, Meta y los santanderes, entre los 450 y 1.500 msnm (Lotero, 1976).

\section{Descripción botánica}

Es un árbol semicaducifolio de porte bajo o arbusto de 3 a $7 \mathrm{~m}$ de altura, con copa esparcida $o$ abierta, formada por ramas que crecen en forma irregular (figura 1a). Los brotes jóvenes crecen en zigzag y son grisáceo-cerosas con muchas lenticelas rugosas. Estos son densamente pubescentes y las partes más antiguas lisas. Todas sus partes son olorosas al triturarlas (Hoyos, 1989).

El árbol presenta un sistema radical bastante superficialy ramificado, pudiendo originar dos o tres pisos o planos de raíces a diferentes niveles, pero poco profundos (Gardiazábal y Rosenberg, 1988).

El anón ramifica cerca a la base y posee un tallo con una corteza externa de color castaño, desde lisa hasta agrietada; la corteza interna es amarillo-claro, algo amarga. La madera es blanda (Hoyos, 1989). 

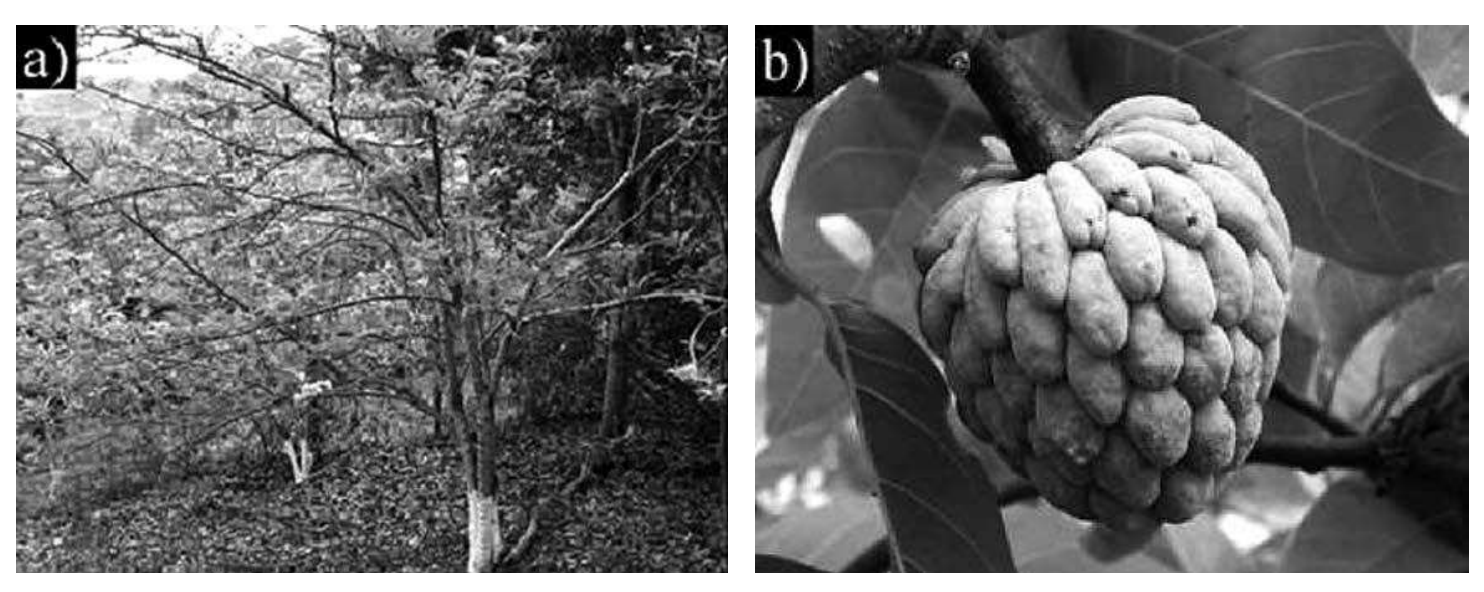

Figura 1. a) Árbol de anón adulto. b) Fruto de anón mostrando el gran número de carpelos que lo conforman (Fotos: E. Guerrero).

Las hojas son sencillas, alternas, elípticas o elíptico-lanceoladas y con margen entero, de 5 a 11 $\mathrm{cm}$ de largo por 2 a $5 \mathrm{~cm}$ de ancho, subagudas en el ápice, cuneiformes en la base; a veces ligeramente asimétricas; grisáceo-cerosas cuando jóvenes, negruzcas al secarse, verde oscuro en la cara superior y verde-azul-pálido en la inferior, lisas en los bordes. Por el haz son glaucas, mientras que por el envés son pubescentes. Los pecíolos tienen una longitud de 5 a $12 \mathrm{~mm}$ y estos son huecos en su unión con el tallo, protegiendo las yemas que continúan su desarrollo cuando las hojas caen (Franciosi, 1992).

Las flores son péndulas, axilares, hermafroditas y por lo general solitarias aunque pueden crecer en grupos de dos a cuatro opuestos a las hojas; fragantes y de coloración verde en la parte externa y crema en la parte interna. Presentan seis pétalos, en dos series: los externos lineal-oblongos, obtusos, de 1 a $3 \mathrm{~cm}$ de largo, carnosos; los pétalos internos son rudimentarios, de más o menos $1 \mathrm{~mm}$ de largo (Hoyos, 1989). El gineceo apocárpico está compuesto por más de 100 carpelos, con ovario súpero y estigma simple. El androceo está formado por más de 100 estambres libres. Las estructuras reproductivas están dispuestas en forma de espiral sobre un receptáculo floral. En la región basal de los pétalos, hay una cavi- dad en la cual se encuentran glándulas secretoras, formando una cámara floral, que sirve como refugio y fuente de alimento para los polinizadores (Gardiazábal y Rosenberg, 1988).

Los frutos son globosos-oviformes, casi de forma acorazonada, de 5 a $12 \mathrm{~cm}$ de diámetro y un peso de 200 a 800 g. Es de color verde-amarillento pero se conocen variedades de color púrpura. Externamente la unión de los carpelos es laxa, con toda su superficie marcadamente prominente, dándole al fruto apariencia tuberculada (figura $1 b)$. La pulpa es blanca o amarillenta entre la unión de los carpelos; los frutos son del tipo sincarpo formados por numerosos pistilos de una flor; cada escama pertenece a un carpelo fecundado. La pulpa es blanco-amarillenta, dulce y aromática, mantecosa, comestible, de agradable sabor (Nakasone y Paull, 1998; Navarro, 2001). Las semillas son oblongas, negro-lustrosas o café-oscuras, de $1,25 \mathrm{~cm}$ de longitud y constituyen entre el 31\% y $41 \%$ del total del fruto y contienen entre 14 y 49\% de aceite (Leal, 1990).

\section{REOUERIMIENTOS AGROECOLÓGICOS}

Según Hoyos (1989), el anón crece desde el nivel del mar hasta más o menos los $1.000 \mathrm{~m}$ de alti- 
tud. A excepción de la chirimoya, las anonáceas no requieren períodos fríos y por el contrario se desarrollan y crecen bien en tierras bajas donde las temperaturas son relativamente estables (George y Nissen, 1987a). Las temperaturas medias más convenientes para el anón varían de 10 a $20^{\circ} \mathrm{C}$ como mínimas y de 22 a $28^{\circ} \mathrm{C}$ como máximas.

Pese a ser propia de climas cálidos es, junto con la chirimoya, la anonácea más resistente al frío (Navarro, 2001); sin embargo, cuando la temperatura desciende bajo $0^{\circ} \mathrm{C}$ provoca la muerte de árboles jóvenes. Los árboles adultos muestran cierta tolerancia a las heladas (Phillips y Campbell, 1994). Por otro lado, las temperaturas mayores a $30^{\circ} \mathrm{C}$ afectan la polinización, aceleran la respiración y reducen la vida poscosecha de los frutos (Leal, 1990).

Higuchi et al. (1998) compararon el efecto de diferentes regímenes de temperaturas entre el día y la noche sobre el crecimiento y la respuesta fisiológica de árboles de anón, encontrándose que a temperaturas día/noche de $30 / 25^{\circ} \mathrm{C}$ la actividad fotosintética es mayor y se ve reflejado en un crecimiento vigoroso de brotes. Asimismo, a estas temperaturas, la concentración de almidón es más alta, la producción total de materia seca es tres veces mayor y la tasa de asimilación de $\mathrm{CO}_{2}$ es dos veces mayor en comparación con plantas que fueron sometidas a temperaturas día/noche de $20 / 15^{\circ} \mathrm{C}$.

La planta soporta fácilmente períodos prolongados de sequía y es probablemente la anonácea más tolerante a esta. Para su desarrollo requiere una precipitación anual entre 750 y $1.200 \mathrm{~mm}$. Durante el verano conviene regarlos de 12 a 15 días. Morton (1987) reporta que en sequías severas, los árboles botan sus hojas y la cáscara de los frutos se endurece produciéndose el rajado de estos cuando llegan las lluvias. El anón se favorece con las lluvias que caen antes de la floración y alcanza una buena producción cuando la humedad del suelo es uniforme, pero, por el contrario, crece y produce poco en áreas donde las lluvias son muy frecuentes (Nakasone y Paull, 1998).

La humedad relativa es uno de los factores climáticos altamente responsables de la polinización; además, los estomas de las hojas de las anonáceas son muy sensibles a cambios en esta. Por esta razón si se quiere aumentar la productividad, es necesario mantener la HR por encima del $60 \%$ sobre todo durante el período de floración mediante microaspersores (Nakasone y Paull, 1998).

La madera blanda de los árboles hace que sean susceptibles a daños por vientos fuertes como rotura y desgarre de ramas; además la epidermis de los frutos es fácilmente dañada por el roce con las ramas (Marler et al., 1994). Por otro lado, los vientos fuertes y secos aceleran la desecación de los estigmas, reduciendo la polinización (Nakasone y Paull, 1998).

Un alto sombreado en árboles vigorosos puede reducir la producción. Un árbol con copa frondosa solo permite la penetración de la luz a la base en un $2 \%$, lo cual es muy poco. En estos casos se recomienda mejorar la penetración de la luz mediante podas y densidades de siembra adecuadas.

Un exceso de radiación solar sobre los frutos causa quemaduras y daños que reducen notablemente la calidad. Para evitar esto se deben proteger los frutos dejando hojas encima de estos. No se conocen respuestas de los árboles al fotoperíodo.

El anón es poco exigente de la clase de suelos, tolerando suelos pobres, pedregosos y con $\mathrm{pH}$ de 7 a 8 de zonas semiáridas; por esta razón se utiliza como portainjerto de otras anonáceas. Crece en un amplio rango de suelos, desde arenosos hasta franco arcillosos, pero las mejores cosechas se obtienen en los suelos fértiles, sueltos y profundos, con pH neutro o ligeramente alcalino y de texturas arenosas o franco arenosas con buen drenaje y aireación, ya que no tolera un nivel freático alto. Además el drenaje es esencial para evitar enfermedades y pudriciones radica- 
les. El crecimiento está en relación directa con el contenido de materia orgánica del suelo (Nakasone y Paull, 1998).

\section{VARIEDADES}

Existe una gran variabilidad genética dentro de esta especie, pero se han desarrollado pocas variedades de importancia comercial. Quizá el 'Seedless Cuban' sea el cultivar más importante, el cual fue desarrollado en Cuba e introducido a la Florida en 1955. Presenta frutos ligeramente malformados, de tamaño medio y con vestigios de semillas no desarrolladas. El sabor es menos atractivo que el de los frutos normales pero es propagado vegetativamente y distribuido como una novedad. Existe otro cultivar cubano que tiene bajo contenido de fibra (Nakasone y Paull, 1998).

Morton (1987), afirma que horticultores hindúes estudiaron la diversidad de anones silvestres y cultivados en diferentes países y reconocieron once tipos: 'Red' (A. squamosa var. Sangareddyiz), 'Red-speckled', 'Crimson', 'Yellow', 'White-stemmed', 'Mammoth' (A. squamosa var. Mammoth), 'Balangar', 'Kakarlapahad', 'Washington', 'Barbados' y 'British Guiana'. Otros cultivares crecen en la Estación Experimental Sabahia, en Egipto: 'Beni Mazar', 'Abd El Razik'.

Se han hecho cruces interespecíficos con el fin de determinar la compatibilidad que existe entre anonáceas y hasta el momento, la atemoya ( $A$. squamosa $\times$ A. cherimola) es el único híbrido desarrollado con importancia comercial (Nakasone y Paull, 1998).

\section{PROPAGACIÓN}

La propagación sexual es el método tradicional a través del cual el anón se ha venido propagando y es el más utilizado por los productores en el municipio de Apulo (Guerrero, 2005). Este método tiene varias desventajas como baja germinación, alta variabilidad genética, el inicio de la cosecha es tardío y las plantas son de mayor altura, lo que dificulta su manejo (Morton, 1987; Cruz, 2002).

Para la recolección de las semillas deben seleccionarse árboles con excelente producción y sanidad; de los que se escogerán los frutos de mejor calidad. Las semillas pierden rápidamente su viabilidad (aproximadamente en 6 meses); razón por la cual estas deben ser sembradas tan pronto como sea posible (Nakasone y Paull, 1998). La siembra debe hacerse en forma horizontal, a $2 \mathrm{~cm}$ de profundidad y a una distancia de $1,5 \mathrm{~cm}$. La germinación se inicia a partir de 30 días (Cruz, 2002).

Varios autores han constatado que las semillas de las anonáceas presentan sustancias inhibidoras de la germinación que provocan latencia lo que, juntamente con un tegumento resistente e impermeable, proporcionan factores antagónicos a una germinación rápida y uniforme (Ratan et al., 1993; Pawshe et al., 1997; Smet et al., 1999). Con respecto a este tema se han realizado algunos trabajos. Lemos et al. (1988) encontraron que la escarificación con lija aumenta la germinación y la velocidad de emergencia en semillas de anón. Colauto et al. (2003) escarificaron las semillas con lija y las sometieron a los siguientes tratamientos por $24 \mathrm{~h}$ : ácido giberélico $\left(\mathrm{GA}_{3}\right)$ a 50 ppm; $\mathrm{GA}_{3}$ a $100 \mathrm{ppm}$; agua a $5^{\circ} \mathrm{C}$ y agua a $30^{\circ} \mathrm{C}$. El tratamiento de $50 \mathrm{ppm} \mathrm{de} \mathrm{GA}_{3}$ fue significativamente superior a los demás tratamientos con $75 \%$ de germinación, en cuanto que 100 ppm de $\mathrm{GA}_{3}$ presentó $44 \%$ y los demás tratamientos tuvieron de $2,5 \%$ a 3,7\%. Del mismo modo, el índice de velocidad de germinación fue mayor para semillas tratadas con $\mathrm{GA}_{3}$ a 50 y 100 ppm; ocurriendo esta entre los 14 y 28 días.

El trasplante a bolsa se hace cuando las plántulas tienen entre 10 a $12 \mathrm{~cm}$ de altura. En el vivero, las plantas duran entre 6 a 8 meses antes de ser pasadas al sitio definitivo (Cruz, 2002).

El otro tipo de propagación que se puede realizar en anón es por la vía asexual o vegetativa me- 
diante la utilización de injertos. Este método es el más recomendable, porque se garantizan plantas con la misma identidad genética, con una mejor producción y calidad de los frutos. Además se facilita el manejo de la plantación (Gardiazábal y Rosenberg, 1988).

De acuerdo con Bezerra y Lederman (1997), dentro de los patrones que pueden ser utilizados en el cultivo del anón, están Annona squamosa L., Annona reticulata L. y Annona glabra L., siendo que el patrón más recomendado es el de la propia especie (A. squamosa L.). En Egipto se utiliza Annona senegalensis como patrón (Morton, 1987). Estos patrones o portainjertos tienen una alta resistencia tanto a la sequía como al exceso de humedad en los suelos (Gardiazábal y Rosenberg, 1988). El anón también puede ser utilizado como patrón para otras especies de anonáceas, como por ejemplo la guanábana y la chirimoya (Bezerra y Lederman, 1997).

Uno de los problemas que presenta la injertación en anonáceas, es que es un método lento de propagación (Bridg, 1992). Según Sanewski (1991), las plantas injertadas pueden producirse en aproximadamente 18 meses, si la propagación se realiza bajo condiciones de invernadero y extenderse a dos años o más, en condiciones de cultivo abierto. Como respuesta a esta problemática, Ferreira et al. (2002) estudiaron el efecto de diferentes concentraciones de $\mathrm{GA}_{3}$ (Progibb) y $\mathrm{GA}_{4+7}$ + fenilmetil-aminopurina (Promalin) en el crecimiento y desarrollo de patrones de A. squamosa; comprobando que la utilización de estos reguladores vegetales afecta positivamente el número de hojas, el diámetro y la longitud del tallo y la producción de materia seca en los portainjertos, reduciendo así el tiempo de injertación.

Las varetas deben tener de 10 a $12 \mathrm{~cm}$ de longitud y deben obtenerse de una rama terminal en estado semileñoso. Es necesario eliminarle las hojas, 7 a 10 días antes de cortarla para activar el crecimiento de las yemas y así tener un mejor prendimiento sobre el patrón (Lazo, 1964; Cruz, 2002).
Los injertos que más éxito han tenido, según Nakasoney Paull (1998) son el de bisel, escudetey púa terminal, con un porcentaje de prendimiento superior al $70 \%$. Después de la injertación, el arbolito debe permanecer tres meses más en vivero antes de trasplantarlo al lugar definitivo (Cruz, 2002).

Otros métodos de propagación asexual en anonáceas como estacas y acodos empleando hormonas de enraizamiento, han tenido poco éxito, ya que se obtienen porcentajes de prendimiento muy bajos (20 a 30\%) y se generan plantas con un pobre sistema radical (Ospina, 1991).

Recientemente se ha venido ensayando con técnicas de micropropagación, mediante el uso del cultivo de tejidos. Las anonáceas son difíciles de propagar in vitro debido a los problemas de contaminación y oxidación del material vegetal (Bridg, 1992); sin embargo, se ha inducido con éxito la organogénesis y la regeneración de plantas in vitro a partir de anteras, endospermo y explantes de hipocótilos y hojas en anón (Rasai et al., 1995; Amin et al., 2002; Nagori y Purohit, 2004).

\section{MANEJO AGRONÓMICO}

\section{Plantación}

El sistema de plantación puede ser en cuadro o en tres bolillo, empleando distancias de $4 \times 4$ ó $5 \times 5 \mathrm{~m}$, para plantas injertadas. El ahoyado depende del tipo de suelo, pero puede ser de $0,30 \times 0,30 \times 0,30 \mathrm{~m}$.

\section{Poda}

Las plantas de anón tienden a formar muchas ramas, por lo que se recomiendan podas de formación regulando la cantidad de ramas principales (Nakasone y Paull, 1998). La chirimoya presenta la particularidad de que en un mismo punto o yema pueden salir cuatro brotes con distinto ángulo de inserción (Gardiazábal y Rosenberg, 1988). Observaciones realizadas en la granja "El Ensueño", parecen confirmar este mismo fenó- 
meno en árboles de anón (Guerrero, 2005). Esto permite elegir yemas que garanticen ramas con un adecuado ángulo de inserción y proporcionen una buena arquitectura a los árboles.

En anonáceas y especialmente en chirimoya se han adoptado dos sistemas principales de formación: en copa abierta o vaso tradicional y en eje central (Franciosi, 1992). Estos sistemas de poda permiten tener una mayor densidad de siembra con árboles de menor altura, facilitando las labores de manejo.

Para la poda de fructificación se debe considerar que las flores del anón surgen en su mayoría en ramas de un año de edad. Por esta razón, la planta necesita emitir ramas nuevas para producir flores. Por tanto, al podar ramas maduras y provocar el deshoje en las plantas, habiendo condiciones ambientales, sobre todo temperaturas por encima de $25^{\circ} \mathrm{C}$, surgen nuevas brotaciones junto con una nueva emisión de flores en esos nuevos brotes (Peres et al., 2002). De esta manera se puede producir durante todo el año, inclusive fuera de la época de cosecha en cultivos bien nutridos y con disponibilidad de riego, lo que constituye una importante alternativa económica para las diversas regiones productoras (Oliveira et al., 2003).

La poda de fructificación se realiza en ramas con más de 3 meses de edad; las cuales, cuando presentan por lo menos $2 / 3$ de su longitud ya madura son cortadas, dejando cerca de 8 a 12 yemas (Kavati y Piza Junior, 1997). Cavalcanti (1993) estima que, en los primeros dos años, son necesarias tres podas por año y en los años siguientes, dos podas espaciadas 6 meses son suficientes en anón.

La poda de renovación se realiza cuando se tengan árboles viejos que hayan decaído su vigor o árboles descuidados. Se debe efectuar una poda fuerte, pero en varias etapas o años para no reducir violentamente la producción; bajando gradualmente la altura de la copa.
La poda de mantenimiento sirve para conservar el equilibrio de la estructura del árbol mediante la eliminación de chupones o brotes que crezcan de manera vertical, así como de ramas que se entrecrucen o se dirijan hacia el suelo. Asimismo, es necesario efectuar después de cada cosecha, una poda sanitaria para eliminar ramas y frutos dañados o con problemas fitosanitarios.

\section{Fertilización}

Cuando se vaya a diseñar el plan de fertilización, se debe dar especial consideración al uso o no de la polinización artificial, ya que los cultivos que se polinizan artificialmente sin la adecuada fertilización, dan buenas producciones durante un año, pero presentan problemas de calidad y calibre de los frutos en los años posteriores, como resultado del agotamiento del huerto (Gardiazábal y Rosenberg, 1988).

Debido al hábito de crecimiento indeterminado que presentan las anonáceas, estas son muy exigentes en nitrógeno y parece que este elemento en exceso no interfiere con la iniciación floral (Nakasone y Paull, 1998). Por el contrario, la falta de $\mathrm{N}$ es la principal causa de restricciones en el crecimiento vegetativo, como lo confirman Sadhu y Ghosh (1976), quienes reportan que plantas de anón deficientes en $\mathrm{N}$ presentan parálisis del crecimiento, sin emisión de ramas. Las hojas son de menor tamaño con tonalidad verde-pálida amarillenta. Surgen manchas de coloración ferruginosa en hojas inferiores y hay abscisión precoz de las mismas. La formación de yemas florales es severamente afectada. El bajo nivel de este nutriente retarda el proceso de floración en más de dos meses; mientras que el alto nivel de $\mathrm{N}$ anticipa la floración en 10 días.

Otro estudio realizado por Lopes et al. (2002) en Fluminense, Brasil, indica que la aplicación de $\mathrm{N}$ y B en anón aumenta el número de frutos y la productividad de las plantas y que el peso promedio del fruto varía en función de las dosis de $\mathrm{N}$ aplicadas. 
El K es otro elemento requerido en grandes cantidades y su deficiencia se caracteriza por la decoloración de los bordes de las hojas, que posteriormente se necrosan (Sadhu y Ghosh, 1976). Los frutos durante su estado de desarrollo requieren grandes cantidades de K; por esta razón, aproximadamente el $60 \%$ de los requerimientos de este elemento deben ser aplicados en este período.

Por su parte, la deficiencia de P provoca reducción del crecimiento e induce la aparición de bandas necróticas de color marrón en las puntas y los márgenes de las hojas (Sadhu y Ghosh, 1976).

Debido al crecimiento vegetativo que se presenta durante el período de desarrollo del fruto, existe una alta competencia de nutrientes, como $\mathrm{B} \mathrm{y} \mathrm{Ca}$ (Sanewski, 1991).

Morton (1987) menciona que la aplicación de fertilizantes comerciales que contengan 3\% de N, 10\% de $\mathrm{P}$ y $10 \%$ de $\mathrm{K}$ incrementa significativamente la floración, la formación de frutos y la cosecha.

En cuanto a la fertilización orgánica, se sabe que las anonáceas responden bien a la aplicación de materia orgánica desde sus primeros estados de crecimiento. La aplicación de fertilizantes orgánicos mejora las condiciones del suelo y facilita el desarrollo de las raíces (Sanewski, 1991; Franciosi, 1992). Morton (1987) recomienda aplicar anualmente de 60 a 80 kg de materia orgánica por árbol.

La fertilización foliar es muy importante, ya que las anonáceas tienen una alta capacidad de absorción de nutrientes por vía foliar (Gardiazábal y Rosenberg, 1988).

En algunos trabajos realizados por Silva (1984) y Oliveira (2000) se han determinado los valores foliares de algunos elementos en anón. Estos autores encontraron que los valores foliares de $\mathrm{N}$ están alrededor de 2,5\%, el P oscila entre 0,15 y $0,17 \%$; mientras que el $\mathrm{K}$ está alrededor de 1,17 y 1,5\%. Los valores foliares de Ca están entre 1,7 y $2,12 \%$ y los de $\mathrm{Mg}$ oscilan entre 0,35 y 0,5\%.
Riego

Debe haber una adecuada humedad en el suelo para fomentar el crecimiento vegetativo y la floración que tiene lugar en las ramas nuevas (Nakasone y Paull, 1998). Es por esta razón que se presume que en plantaciones con riego constante se podrían obtener cosechas durante todo el año.

No se conocen datos exactos sobre los usos consuntivos de esta especie, pero se menciona que su híbrido atemoya puede necesitar aproximadamente $1.440 \mathrm{~L}$ por árbol cada 4 semanas durante el período de bajo crecimiento y de 500 a 750 L por árbol cada 3 a 5 días durante la floración, cuajado y crecimiento del fruto (Sanewski, 1991).

El estrés hídrico debe ser prevenido durante la floración, cuajado y desarrollo del fruto, ya que el fruto es más sensible que las hojas a la falta de agua. La adecuada humedad del suelo y la alta HR durante el período de floración pueden prolongar la receptividad del estigma, favoreciendo la polinización, así como el cuajado y crecimiento de los frutos. La HR se puede incrementar, mediante la instalación de microaspersores debajo de la copa de los árboles (Nakasone y Paull, 1998).

\section{INDUCCIÓN FLORAL}

El conocimiento de la morfología floral y la biología de floración, es imprescindible cuando se tiene en mente el cultivo racional y comercial de anonáceas (Kavati, 1992; Bezerra y Lederman, 1997). La floración del anón está fuertemente asociada al crecimiento vegetativo, con mayor producción de flores localizada en la región basal de las ramas recién emergidas (George y Nissen, 1987b).

La poda de verano ha demostrado potencial para aumentar la producción de algunos cultivares de anón (Batten, 1983). George y Nissen (1987b), comprobaron que el deshoje realizado con urea 
y ácido cloroetilfosfónico (Ethrel), en pre-brotación y una poda de verano fueron más efectivas para anticipar la floración en relación al control, observando que la poda causó un aumento de la producción, en atemoya.

En el estado de Pernambuco, Brasil, Melo et al. (2000) llevaron a cabo un ensayo en un cultivo de anón para inducir la brotación de yemas y la floración realizando dos tipos de deshoje (manual y químico) y aplicando diferentes dosis de dos inductores de brotación (Dormex y Ethrel). Los mejores resultados se obtuvieron con el deshoje manual en $50 \%$ de la planta y la pulverización con cianamida hidrogenada (Dormex) al $1,5 \%$. Estos resultados demuestran que el deshoje y la aplicación de inductores de brotación pueden utilizarse como una alternativa válida para uniformizar e incrementar la floración y facilitar las labores de manejo del cultivo, como la polinización artificial.

\section{POLINIZACIÓN}

Desde que emerge la yema floral, esta se desarrolla permaneciendo cerrada durante aproximadamente 30 días. Una vez la flor ha alcanzado el tamaño definitivo, el ciclo de apertura se produce en tres fases consecutivas (Farré et al., 1999).

En la primera fase llamada prehembra, los pétalos comienzan a separarse por su extremo pero no por su base. En este estado la flor es receptiva, pero no puede ser polinizada por insectos y dura de 6 a 15 h. En la segunda fase, la fase hembra, los pétalos continúan su separación, permitiendo la entrada de pequeños insectos polinizadores a la masa estigmática. La duración de esta fase es aproximadamente 26 a $27 \mathrm{~h}$. Los estigmas son receptivos durante todo el período, excepto durante las tres últimas horas. Finalmente durante la tercera fase llamada macho, los pétalos se separan totalmente en 20 a 30 min coincidiendo con la separación y apertura de los estambres. La transición al estado macho ocurre, tanto si la flor continúa en el árbol como si es separada del mismo. Cuando las temperaturas son altas el ciclo se acorta, alargándose cuando son bajas (Farré et al., 1999).

En un mismo árbol o inclusive en la totalidad del huerto puede coincidir una sola fase de apertura floral el mismo día, o puede presentarse un traslape de fases (Farré et al., 1999).

Uno de los principales problemas que afectan el rendimiento y la calidad de los frutos de anón, es el bajo índice de polinización de las flores y la consecuente producción de frutos. A pesar de ser morfológicamente perfectas, las flores de las anonáceas presentan dicogamia protogínica, fenómeno por el cual la maduración de los carpelos acontece antes de la maduración de los estambres, inviabilizando la autofecundación (Campos et al., 2004). Este mecanismo de dioecia temporal ha sido probablemente desarrollado por estas especies para favorecer la polinización cruzada entre distintos árboles en estado silvestre (Farré et al., 1999).

Aunque el anón produce una gran cantidad de flores en cada cosecha, se estima que solamente cerca de 3 a $5 \%$ se convierten en frutos. Además Fioravanço y Paiva (1994) observaron que los frutos son, en su mayoría, rechazados por pequeños o malformados, posiblemente por el bajo número de carpelos polinizados.

Como respuesta a este problema se ha estudiado el método de polinización artificial. Los beneficios de esta técnica en cultivares de chirimoya, anón e híbridos de atemoya han sido destacados en diversos trabajos. Cogez y Lyannaz (1996) obtuvieron $90 \%$ de cuajado de frutos de anón con la técnica de polinización manual, comparado con $1 \%$ bajo condiciones naturales. También se obtuvo un incremento sustancial del peso de la fruta proveniente de polinización manual (17 a 62\%).

En un ensayo realizado sobre polinización en el municipio de Apulo (Cundinamarca) se obtuvo 
$87 \%$ de frutos cuajados empleando la polinización artificial, mientras que con polinización natural solo se obtuvo el $15 \%$ de frutos cuajados y con la autopolinización no se obtuvo ningún fruto; indicando que efectivamente esta no ocurre en anón. Estos resultados confirman las ventajas de la polinización artificial. Además de haber obtenido un alto porcentaje de frutos cuajados, estos tuvieron una forma casi perfecta comparado con aquellos frutos provenientes de la polinización natural (Guerrero, 2005).

Las flores de las anonáceas son polinizadas por coleópteros de las familias Nitidulidae, Staphylinidae, Chrysomelidae, Curculionidae o Scarabeidae (Chatrou, 1999); la polinización por trips también se presenta, pero es poco significativa (Momose et al., 1998). Estos insectos son atraídos por el olor a fruta fermentada de las flores y algunas especies se alimentan ocasionalmente de estas. Los polinizadores entran en la flor cuando está en su fase femenina en horas de la mañana y se mantienen inactivos en la base de los pétalos, caminando sobre los estambres y estigmas. Cuando las flores entran en su etapa masculina, se dispersan cubiertos de polen hacia otras flores (Nadel y Peña, 1994).

\section{MANEJO DE ARVENSES}

En el cultivo del anón, las arvenses provocan los mayores inconvenientes durante las etapas de vivero y establecimiento. La época crítica de competencia se da en los períodos de máxima división y elongación celular, la emisión de yemas, floración y fructificación, ya que en estos períodos son mayores las exigencias de nutrientes y humedad del suelo (Linares, 1991).

El control puede ser manual, mecánico, con coberturas vivas o inertes, con el uso de herbicidas o con la combinación de métodos haciendo un manejo integrado. No se debe dejar completamente desnudo el suelo y hay que tener en cuenta que las labores de control deben hacerse su- perficialmente para no causar daños al sistema radical de las plantas (Guerrero, 2005).

\section{MANEJO DE PLAGAS}

El anón es muy vulnerable al ataque de plagas que reducen la producción, causan una depreciación comercial del producto y afectan la longevidad productiva de los cultivos. Esto ha venido desestimulando y limitando el establecimiento de nuevos cultivos comerciales (Cardoso et al., 2004).

Las plagas más importantes que se encontraron en la zona de estudio corresponden a perforadores del fruto, insectos de hábito chupador y algunos comedores de follaje (Guerrero, 2005).

Dentro del grupo de plagas que causan daño a los frutos se encuentra la avispa perforadora de las semillas (Hymenóptera: Eurytomidae) (Bephratelloides maculicollis Cam.) y la polilla perforadora del fruto (Lepidóptera: Stenomatidae) (Cerconota anonella Sepp.). Los frutos atacados se momifican debido a que las perforaciones dejadas por estas plagas facilitan la entrada y el establecimiento de hongos patógenos. El nivel de daño causado por estos insectos puede alcanzar mucho más del 50\% de los frutos (Escobar y Sánchez, 1992).

Para el manejo y control de estas plagas se recomienda la recolección, tanto del suelo como del árbol, de los frutos afectados para su posterior destrucción. Otra práctica recomendada es la de proteger los frutos con bolsa plástica, papel o polietileno, en los estados iniciales de desarrollo. Las bolsas deben cubrir el fruto durante todo su desarrollo, deben estar abiertas en la base y tener microperforaciones para permitir el intercambio gaseoso del fruto y la salida del agua, pues un exceso de humedad causa alta pudrición de los frutos. Se recomienda asperjar los frutos con insecticidas y fungicidas en pequeñas dosis antes del embolse (Escobar y Sánchez, 1992). 
Se ha determinado que un buen control de malezas causa disminución en la presencia de estas plagas.

En cuanto a los enemigos naturales, se encuentran los parasitoides Apanteles stenomae y Xuphosomella sp.

Las medidas de control químico son difíciles, si se tiene en cuenta que estas plagas están protegidas la mayor parte del tiempo dentro del fruto; además el uso de insecticidas destruye los enemigos naturales y los insectos polinizadores. Para el control de la polilla, además, se utilizan mecheros o trampas de luz, especialmente en las épocas de mayor presencia de los adultos.

Otro complejo de plagas importante es el de los chupadores, dentro de los que se encuentran los áfidos o pulgones, la escama blanda de la familia Coccidae (Phylephedra sp.), la escama globosa (Saissetia sp.) y la cochinilla harinosa de la familia Pseudococcidae (Guerrero, 2005). Como plagas de tipo masticador se encuentran larvas de lepidópteros y algunos coleópteros (Escobar y Sánchez, 1992).

\section{MANEJO DE ENFERMEDADES}

Dentro de las enfermedades más comunes y limitantes que afectan al anón en el municipio de Apulo se encuentran la antracnosis (Colletotrichum gloeosporioides) atacando flores, frutos y hojas y la mancha blanca del follaje (Cercospora annonae). Estas enfermedades aumentan su incidencia y severidad en épocas de lluvia, con temperaturas altas y estables y humedad relativa elevada.

Se reporta que el anón es atacado por otras enfermedades en diferentes partes del mundo, las cuales, dependiendo de las condiciones climáticas de cada región, pueden ser más o menos limitantes dentro del cultivo (Ploetz, 2003).

\section{COSECHA}

El anón comienza a producir a la edad de tres años y declina después de los 12 a 15 años. En promedio un árbol adulto produce entre $100 \mathrm{y}$ 150 frutos al año con un peso que oscila entre 120 y 230 g (Rajput, 1985).

La decisión del momento óptimo de cosecha es algo crítico y desafortunadamente no se conocen, ni se han establecido índices de madurez adecuados para el anón. Además, los frutos no maduran todos al mismo tiempo y la época de la recolección varía según la variedad y las condiciones climatológicas del lugar donde se efectúa el cultivo. Los métodos visuales, basados en el cambio de coloración de la piel y forma de las escamas del fruto, son los que más se emplean en la actualidad para decidir el momento de cosecha (Gardiazábal y Rosenberg, 1988). El anón se cosecha cuando los frutos toman una coloración verde amarillenta entre los carpelos, los cuales se separan posteriormente dejando al descubierto porciones de la pulpa (Morton, 1987). Otro índice utilizado por los productores es el cambio de coloración de las semillas, que pasan de un color marrón claro a un color casi negro.

Cuando se emplea la técnica de la polinización artificial se puede programar la época de cosecha, teniendo en cuenta que en promedio los frutos tardan entre 110 y 120 días desde la floración hasta la madurez de cosecha (Pal y Sampath, 1995; Cogez y Lyannaz, 1996).

Estos criterios generalmente no son confiables para indicar la madurez apropiada, ya que no se consideran los constituyentes químicos que determinan el sabor. Por esta razón se deben considerar, además de los parámetros físicos, los cambios químicos y fisiológicos del fruto.

En un estudio realizado en la India Pal y Sampath (1995), encontraron que los frutos duran aproximadamente $120 \pm 5$ días para alcanzar la 
madurez de cosecha y otros 2 a 5 días para llegar a la madurez de consumo a temperaturas de $28 \pm 3^{\circ} \mathrm{C}$. El peso y tamaño de los frutos se incrementa hasta alcanzar la madurez de cosecha. El patrón de crecimiento de los frutos fue de tipo doble sigmoidal. La densidad del fruto disminuye hasta la madurez de cosecha y luego incrementa. Beerh et al. (1983) reportan que el porcentaje de sólidos solubles oscila entre $20,6 \%$ y $28,0 \%$ en frutos maduros de diferentes cultivares de anón.

Durante la maduración, la acidez total aumenta, al igual que los azúcares reductores y no reductores provenientes de la conversión del almidón que disminuye. Se considera que la relación azúcar/ácido y el color amarillento entre los carpelos son criterios aceptables para determinar el punto óptimo de cosecha (Pal y Sampath, 1995). El fruto del anón tiene un alto contenido de proteína y azúcares. Es una fuente importante de minerales como Fe, Ca y P y vitaminas como el ácido ascórbico (tabla 1).

El fruto de anón es muy delicado y se debe cosechar cuando el ambiente está seco, sin lloviznas o elevada HR. Hay que regular el riego durante la cosecha, ya que los excesos de agua aumentan la susceptibilidad de la fruta al manipuleo y se deben cosechar las frutas cortando a ras el pedúnculo, empleando tijeras de podar. También se recomienda cosechar en horas de la mañana cuando el fruto tiene temperaturas relativamente bajas (Gardiazábal y Rosenberg, 1993).

\section{MANEJO POSCOSECHA}

Debido a lo delicado del manejo de estos frutos, que fácilmente desprenden sus "escamas" y por la corta vida poscosecha, el consumo es básicamente local y muy difícil su exportación (Hoyos, 1989).

En el estudio realizado por Pal y Sampath (1995), se encontró que el patrón de respiración de $A$. squamosa durante la maduración poscosecha fue
Tabla 1. Valor nutricional en $100 \mathrm{~g}$ de pulpa (según Morton, 1987).

\begin{tabular}{|l|r|}
\hline Componentes & Concentración \\
\hline Calorías & $88,9-95,7$ \\
\hline Humedad & $69,8-75,18 \mathrm{~g}$ \\
\hline Grasa & $0,26-1,10 \mathrm{~g}$ \\
\hline Carbohidratos & $19,16-25,19 \mathrm{~g}$ \\
\hline Proteína & $1,53-2,38 \mathrm{~g}$ \\
\hline Fibra & $1,14-2,50 \mathrm{~g}$ \\
\hline Cenizas & $0,55-1,34 \mathrm{mg}$ \\
\hline Fósforo & $23,6-55,3 \mathrm{mg}$ \\
\hline Calcio & $19,4-44,7 \mathrm{mg}$ \\
\hline Hierro & $0,28-1,34 \mathrm{mg}$ \\
\hline Triptofano & $9-10 \mathrm{mg}$ \\
\hline Metionina & $7-8 \mathrm{mg}$ \\
\hline Lisina & $54-69 \mathrm{mg}$ \\
\hline Caroteno & $5-7 \mathrm{U} . \mathrm{l}$. \\
\hline Tiamina & $0,10-0,13 \mathrm{mg}$ \\
\hline Riboflavina & $0,113-0,167 \mathrm{mg}$ \\
\hline Niacina & $0,654-0,931 \mathrm{mg}$ \\
\hline Ácido ascórbico & $34,7-42,2 \mathrm{mg}$ \\
\hline
\end{tabular}

de tipo climatérico, presentándose el pico climatérico a los 3 o 4 días después de la cosecha.

Las condiciones de almacenamiento recomendadas para anón están entre 15 y $20^{\circ} \mathrm{C}$, con una HR de 85 a $90 \%$. El preenfriamiento de los frutos es esencial para ayudar a prolongar la vida poscosecha (Broughton y Guat, 1979; Nakasone y Paull, 1998).

El anón presenta una vida corta en poscosecha, debido a una rápida pérdida de la firmeza de la pulpa que está asociada con la producción y la acción del etileno. Benassi et al. (2003) evaluaron diferentes concentraciones de 1- metilciclopropeno (1-MCP) que es un antagonista del etileno durante $12 \mathrm{~h}$ a $25^{\circ} \mathrm{C}$. Enseguida los frutos fueron almacenados a $25^{\circ} \mathrm{C}$ durante 4 días. Se encontró que los frutos tratados con $810 \mathrm{~nL} \cdot \mathrm{L}^{-1}$ de 1-MCP presentaron mayor firmeza de la pulpa, mientras los tratados con 30 y $90 \mathrm{~nL} \cdot \mathrm{L}^{-1}$ maduraron más rápido que los tratados en mayores concentraciones. 


\section{COMERCIALIZACIÓN}

El anón se comercializa generalmente como fruta fresca en los lugares donde se produce. En Apulo y en los demás municipios productores, los campesinos venden el anón en cajas de madera a intermediarios. Quienes luego ofrecen los frutos en las principales ciudades del país y una vez allí, el anón es distribuido a granel.

Este sistema de comercialización es totalmente deficiente ya que existen grandes diferencias en la conformación del precio a través de los diferen- tes eslabones de la cadena, siendo los productores los que reciben la menor retribución económica.

Guerrero (2005) encontró que en Colombia no existen normas técnicas de calidad, ni estadísticas claras de los volúmenes de producción. Esto hace que haya una desorganización a nivel interno y se dificulte aún más la comercialización. Además, no se conoce la dinámica que tiene el producto en los mercados internacionales. Es necesario estimar la demanda y la oferta real y potencial con el fin de incursionar positivamente en estos mercados.

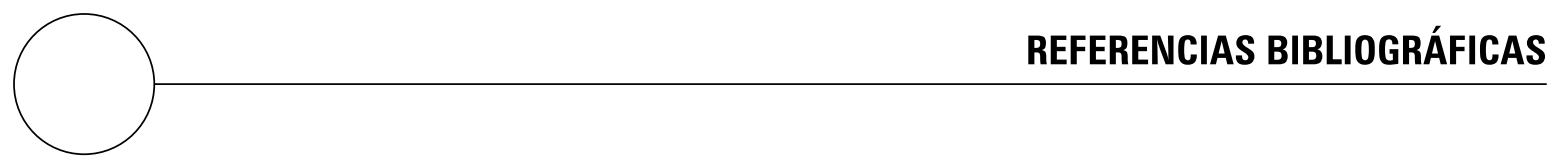

Amin, M.; K. Nahar; F. Ahmed y S. Ahmad. 2002. Micropropagation of Annona squamosa Linn. using explants (shoot tip and node) of field grown mature plants. Pakistan J. Biol. Sci. 5(4), 394-397.

Batten, D. 1983. Custard apple pruning and yield. En: Research report 1982-83, Fruit Research Station, Astonville. pp. 1-16.

Beerh, O.; N. Giridhar y B. Raghuramaiah. 1983. Custard apple (Annona squamosa). Indian Food Packer 37(3), 77-81.

Benassi, G.; G. Correa; R. Kluge y A. Jacobino. 2003. Shelf life of custard apple treated with 1-methylciclopropene an antagonist to the ethylene action. Braz. Arch. Biol. Techn. 46(1), 115-120.

Bezerra, J. y I. Lederman. 1997. Propagação vegetativa de anonáceas por enxertia. pp. 61-67. En: São José, A.; I. Souza; O. Morais y T. Rebouças (eds.). Anonáceas, produção e mercado (pinha, graviola, atemóia e cherimólia). Vitória da Conquista, Brasil. 306 p.

Bridg, H. 1992. Alternativas para la propagación de chirimoya (Annona cherimola Mill.). Tesis de especialización en frutales de clima frío. Facultad de Ciencias Agropecuarias, Universidad Pedagógica y Tecnológica de Colombia, Tunja.

Broughton, N. y T. Guat. 1979. Storage conditions and ripening of the custard apple Annona squamosa L. Sci. Hort. (1), 73-82.

Campbell, C. y R. Phillips. 1994. The sugar apple. Horticultural Sciences Department, Florida Cooperative
Extension Service, Institute of Food and Agricultural Sciences, University of Florida.

Campos, R.; E. Lemos; J. Oliveira; F. Pereira; A. Dias y P. Barros. 2004. Polinização natural, manual e autopolinização no pegamento de frutos de pinheira (Annona squamosa L.). Revista Brasileira de Fruticultura 26(2), 261-263.

Cardoso, J.; F. Seixas; J. Vidal y R. Martins. 2004. Influência da poda e da lâmina de água de irrigação na produção e na incidência da podridão-seca em ateira. Boletim de Pesquisa e Desenvolvimento 15. EMBRAPA, Brasil. 17 p.

Cavalcanti, R. 1993. A cultura da pinha (Annona squamosa L.). En: Encontro estadual de fruticultura 1. Cruz das Almas. EMBRAPA/CNPMF, Brasil. 159 p.

Cogez, X. y J. Lyannaz. 1996. Manual pollination of sugar apple (Annona squamosa). Fruits 49(5-6), 359-360.

Colauto, N.; I. Massanori y C. Vieira. 2003. Superação da dormência em sementes de atemóia e fruta-do-conde. Revista Brasileira de Fruticultura 25(2), 305-308.

Chatrou, L. 1999. Las annonaceae y el proyecto annonaceae: una breve revisión de su estado actual. Acta Hort. 497, 51-57.

Cruz, E. 2002. Cultivo de Anona. Boletín Técnico No. 7. Centro Nacional de Tecnología Agropecuaria y Forestal, CENTA, El Salvador. 20 p.

Escobar, W. y L. Sánchez. 1992. Control de plagas y enfermedades del guanábano. Boletín de sanidad vegetal 
07. ICA. Separata del manual Fruticultura Colombiana Guanábano. Produmedios, Bogotá, pp. 61-78.

Farré, J.; J. Hermoso y E. Guirado. 1999. Técnicas de cultivo del chirimoyo en España. Acta Hort. 497, 105-118.

Ferreira, G.; P. Erig y E. Moro. 2002. Produção do portaenxerto (Annona squamosa L.) com o uso de reguladores vegetais. Revista Brasileira de Fruticultura $24(3), 637-640$

Fioravanço, J. y M. Paiva. 1994. Tratos culturais. En: Fioravanço, J. y Paiva, M. (eds.). Fruticultura, cultivo das anonáceas: Ata, cherimóia e graviola. Porto Alegre, Brasil. pp. 62-77.

Franciosi, R. 1992. El cultivo del chirimoyo en el Perú. Primera edición. Ediciones Fundeagro, Perú. 108 p.

Gardiazábal, F. y M. Rosenberg. 1988. Cultivo del chirimoyo. Facultad de Agronomía, Universidad Católica de Chile, Chile. 112 p.

George, A. y R. Nissen. 1987a. Propagation of Annona species: A review. Sci. Hort. 33, 75-85.

George, A. y R. Nissen. 1987b. Effects of cincturing, defoliation and summer pruning on vegetative growth and flowering of custard apple (Annona cherimola $\mathrm{X}$ Annona squamosa) in subtropical Queensland. Aust. J. Exp. Agr. 27, 915 - 918.

Guerrero, E. 2005. Estudio sobre el manejo integrado del cultivo del anón (Annona squamosa L.) en el municipio de Apulo, Cundinamarca. Trabajo de grado. Facultad de Agronomía. Universidad Nacional de Colombia, Bogotá. 54 p.

Higuchi, H.; N. Utsunomiya y T. Sakuratani. 1998. Effects of temperature on growth, dry matter production and $\mathrm{CO} 2$ assimilation in cherimoya (Annona cherimola Mill.) and sugar apple (Annona squamosa L.) seedlings. Sci. Hort. 73, 89-97.

Hoyos, J. 1989. Frutales en Venezuela. Sociedad de Ciencias Naturales La Salle. Monografía 36, pp. 35-48.

Kavati, R. 1992. Cultivo da atemóia. pp. 39-70. En: Donadio, L.; A. Martins y J. Valente (eds.). Fruticultura tropical. FUNEP, Jaboticabal, Brasil.

Kavati, R. y C. Piza Junior. 1997. Formação e manejo do pomar de fruta-do-conde, atemóia e cherimólia. pp.75-83. En: São José, A.; I. Souza; O. Morais y T. Rebouças (eds.). Anonáceas, produção e mercado (pinha, graviola, atemóia e cherimólia). Vitória da Conquista, Brasil. 306 p.

Lazo, F. 1964. Anonáceas su propagación. Agencia para el Desarrollo Internacional (AID). Misión económica de los Estados Unidos. 4 p.
Leal, F. 1990. Sugar Apple. pp. 149-158. En: Nagy, S., P. Shaw, y W. Wardowski (eds.). Fruits of tropical and subtropical origin. Composition, properties and uses. Florida Science Source, Lake Alfred, Florida.

Lemos, E.; R. Cavalcanti; A. Carrazoni y T. Lobo. 1988. Germinação de sementes de pinha submetidas a tratamentos para quebra de dormência. En: Congresso Brasileiro de Fruticultura 9(2), 675-678.

Linares, H. 1991. Manejo de malezas en el guanábano. pp. 71-87. En: Asociación de Ingenieros Agrónomos del Sector Frutícola, ASIAFRUT (eds.). Primer curso nacional de guanábana. Mayo de 1991. Ibagué.

Lopes, S.; A. Cordeiro; P. Oliveira; P. Monnerat y C. Marinho. 2002. Produtividade da cultura da pinha (Annona squamosa L.) em função de níveis de adubação nitrogenada e formas de aplicação de boro. Revista Brasileira de Fruticultura 24(2), 543-546.

Lotero, A. 1976. Géneros de la flora de Colombia III, "Anonaceae". Trabajo de grado. Departamento de Biología, Universidad Nacional de Colombia, Bogotá.

Marler, J.; A. George; R. Nissen y P. Andersen. 1994. Miscellaneous tropical fruits-annonas. pp. 200-206. En: Schaffer, B. y P. Andersen (eds.). Handbook of environmental physiology of fruit crops. Vol. II, Subtropical and tropical crops. CRC Press Boca Raton, Florida.

Melo, M.; N. Gonzaga; J. Bezerra; I. Lederman y J. Assis. 2000. Efeito de indutor de brotação associado a desfolha química e manual na indução floral de pinheiras (Annona squamosa L.). EMBRAPA. Brasil. En: http://www.ufpel.tche.br/sbfruti/anais_xvii cbf/ fisiologia/852.htm; consulta: abril de 2005.

Momose, J.; T. Nagamitsu y T. Inoue. 1998. Thrips crosspollination of Popowia pisocarpa (Annonaceae) in a lowland dipterocarp forest in Sarawak. Biotropica $30,444-448$.

Morton, J. 1987. Sugar apple (Annona squamosa). pp. 69-72. En: Morton, J. (ed.). Fruits of warm climates. Creative Resources Systems, Miami.

Nadel, H. y J. Peña. 1994. Identity, behavior, y efficacy of nitidulid beetles (Coleoptera: Nitidulidae) pollinating commercial Annona species in Florida. Environ. Entomol. 23, 878-886.

Nagori, R. y S. Purohit. 2004. In vitro plantlet regeneration in Annona squamosa through direct shoot bud differentiation on hypocotyl segments. Scientia Hort. 99, 89-98.

Nakasone, H. y R. Paull. 1998. Tropical fruits. Crop production science in horticulture series. $C A B$ International, Londres. pp. 45-75. 
Navarro, J. 2001. Guía de las frutas cultivadas. Identificación y cultivo. Mundi-Prensa, Madrid. 224 p.

Oliveira, N. 2000. Resposta da cultura da pinha (Annona squamosa L.) a níveis de adubação NPK. Disertación (Maestría) - Universidad Estatal de São Paulo, Brasil. $56 \mathrm{p}$.

Oliveira, N.; S. Naomi; T. Hojo; A. Silveira; A. Rebouças y I. Bôas, 2003. Influência da poda de produção em ramos de diferentes diâmetros no desenvolvimento vegetativo e reprodutivo da pinheira (Annona squamosa L.). Revista Brasileira de Fruticultura 25(1), 100-103.

Ochse, J.; M. Soule; M. Dijkman y G. Wehlburg. 1971. Tropical and subtropical agriculture. Editorial Limusa-Wiley, México. 760 p.

Ospina, R. 1991. Métodos de propagación en guanábana (Annona muricata L.). pp. 13-22. En: Asociación de Ingenieros Agrónomos del Sector Frutícola, ASIAFRUT (eds.). Primer curso nacional de guanábana. Mayo de 1991, Ibagué. 227 p.

Pal, D. y K. Sampath, 1995. Changes in the physico-chemical and biochemical compositions of custard apple (Annona squamosa L.) fruits during growth, development and ripening. J. Hort. Sci. 70(4), 569-572.

Pawshe, Y.; B. Patil y L. Patil. 1997. Effect of pregermination seed treatment on the germination and vigour of seedlings in custard apple (Annona squamosa L.). Ann. Plant Physiol. 11(2), 150-154.

Peres, L.; M. Sudo-Martelleto; C. Ide y S. Pereira. 2002. Épocas de poda de produção em pinheira (Annona squamosa L.) no município de Miracema, Rio de Janeiro. PESAGRO-RIO. Estación Experimental de Macaé. En: http://www.ufpel.tche.br/sbfruti/anais xvii_cbf/fitotecnia/189.htm;consulta:juniode2005.

Pinto, A. y P. Genú. 1984. Contribuição ao estudo técnico científico da graviola (Annona muricata L.). pp. 529-546. En: Congresso Brasileiro de Fruticultura, 7 , Florianópolis, Sociedade Brasileira de Fruticultura.

Ploetz, R. 2003. Diseases of atemoya, cherimoya, soursop, sugar apple and related fruit crops. pp. 21-34. En: Ploetz, R. (ed.). Diseases of tropical fruit crops. CABI Publishing, Londres.

Rajput, C. 1985. Custard apple. pp. 479-486. En: Bose, T. (ed.). Fruits of India Tropical and Subtropical. Department of Horticulture, India.

Rasai, S.; A. George y A. Kantharajah. 1995. Tissue culture of Annona spp. (cherimoya, atemoya, sugar apple and soursop): A review. Scientia. Hort. 62, 1-14.

Ratan, P.; S. Reddy y Y. Reddy. 1993. Influence of water soaking on Annona squamosa L. seed germina- tion and subsequent seedling growth. South Indian Hort. 41(3), 171-173.

Sadhu, M. y S. Ghosh, 1976. Effects of different level of nitrogen, phosphorus and potassium on growth, flowering, fruiting and tissue composition of custard apple (Annona squamosa). Indian Agr. 20(4), 297-301.

Sanewski, G. 1991. Custard Apples - Cultivation and crop protection. Information Series QI0031. Queensland Departament of Primary, Industry, Brisbane. 103 p.

Silva, A. 1984. Conteúdo de nutrientes por ocasião da colheita em diversos frutos da região do nordeste. pp. 326-340. En: Congresso Brasileiro de Fruticultura 7. Florianápolis.

Smet, S.; P. Damme; X. Scheldeman y J. Romero. 1999. Seed structure and germination of cherimoya ( $A n$ nona cherimola Mill.). Acta Hort. 497, 269-278.

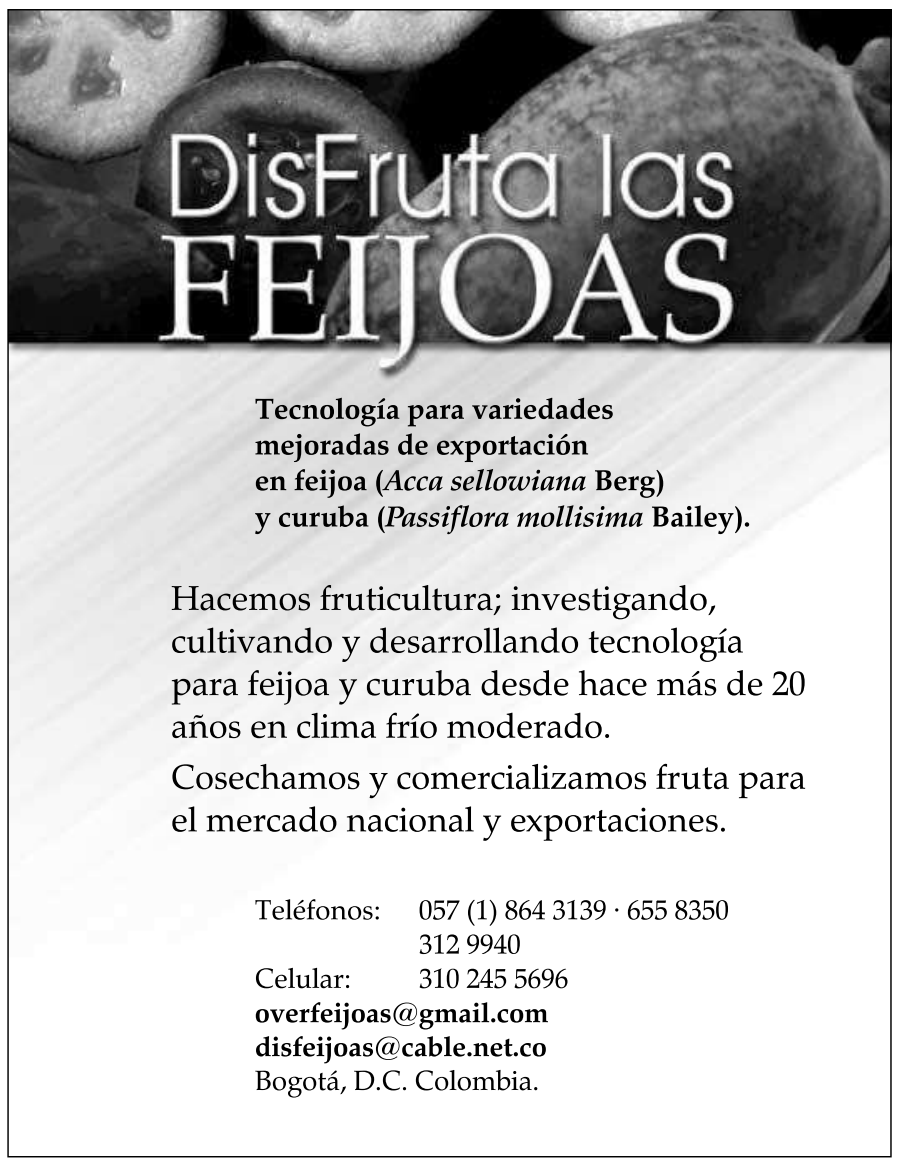

